\title{
Rapid label-free DNA analysis in picoliter microfluidic droplets using FRET probes
}

\author{
Albert Tsung-Hsi Hsieh · Patrick Jen-Hao Pan • \\ Abraham Phillip Lee
}

Received: 29 October 2008/Accepted: 30 December 2008/Published online: 11 February 2009

(C) The Author(s) 2009. This article is published with open access at Springerlink.com

\begin{abstract}
We report a novel microfluidic system that is capable of rapidly detecting DNA and its mutants in microfluidic droplets, in addition to elucidating the dynamic hybridization process. This microfluidic picoliter droplet analysis system is able to overcome the limitations of conventional analytical techniques that utilize immobilized sensing probes on a substrate. Molecular beacon (MB), a fluorescence resonance energy transfer (FRET) molecule, was used as the DNA sensing probe in picoliter droplets. The MB-DNA duplex formation process was analyzed by the change in FRET signal, which was acquired by the time-resolved method: converting distance traveled to hybridization time. This technique demonstrates the ability to detect presence of target nucleic acids within few seconds, multiplex DNA samples in microdroplet, and distinguish single nucleotide polymorphisms. It is promising for analyzing biomolecules or reactions, such as mRNA, cells, enzymatic activity, and protein folding whose analysis requires rapid mixing and small volume.
\end{abstract}

Keywords Microdroplet - Molecular beacon - FRET . DNA analysis · Time-resolved · SNP

A. T.-H. Hsieh · P. J.-H. Pan · A. P. Lee $(\square)$

Department of Biomedical Engineering,

University of California, Irvine, 3120 Natural Sciences II, Irvine, CA 92697-2715, USA

e-mail: aplee@uci.edu

A. T.-H. Hsieh

e-mail: tsung@uci.eu

\section{Introduction}

DNA hybridization is extensively applied in medicine for diagnosing genetic diseases and gene sequencing (Livak 1999; Syvanen 2001). Analysis of DNA sequences with single nucleotide polymorphism (SNP) is one of the major methods for diagnosing genetic diseases. Microarray (Lipshutz et al. 1999; van't Veer et al. 2002), quartz crystal microbalance (QCM) (Caruso et al. 1997; Okahata et al. 1998) and surface plasmon resonance (SPR) (Thiel et al. 1997; He et al. 2000) are examples that have been developed to analyze DNA hybridization, sequencing and SNP. However, these methods are often carried out in bulk solutions with immobilized sensing probes on the substrate surface, which is dependent on the passive diffusion of DNA samples toward the sensing probes. The limited solid-liquid diffusion greatly hinders the hybridization rate of target DNA and sensing probe. Thus, the hybridization time for DNA and immobilized-probe normally takes from several hours to overnight (Peterson et al. 2001; Jobs et al. 2002). Additionally, conditions of sensing probes, such as the random immobilization density, electrostatic hindrance and the length of DNA probes on the substrate will all compromise the precision of dynamic readings (Steel et al. 2000; Peterson et al. 2001; Wong et al. 2005). Moreover, the stringent rinsing processes are also normally required after the hybridization process to remove non-specific binding. It further increases the whole process time. Therefore, it is important to develop a DNA analysis assay which is an easy, faster, accurate and low sample consumption method for multiplex DNA analysis.

Because of the small dimensions of the microfluidic system, the flows with low Reynolds number are always laminar. The diffusion distance was also greatly reduced in microfluidic channel. These unique intrinsic properties of 
microfluidic system contribute to the easily control of liquid behavior in microfluidic channel. Therefore, microfluidic based techniques were applied to overcome the slow hybridization problem caused by the diffusion-limited solid-liquid reaction, and further accelerate nucleic acid hybridization (Sanders and Manz 2000; Lagally et al. 2001; Sia and Whitesides 2003). Mixing in laminar flow system occurs by diffusion alone, which can result in unacceptable long mixing times (Kamholz et al. 1999; Liu et al. 2000). Moreover, uneven dispersion of DNA samples in solution and contamination on microfluidic channel surfaces can spoil accurate quantitative analysis. The DNA sample dispersion in continuous microfluidic system hinders the application of this technique for in situ multiplex DNA sample detection, and different concentrations of DNA sample analysis. Surface treatment is usually required to avoid DNA contaminants in the microfluidic channel.

The monodispersed picoliter microfluidic droplet generation system developed by our group and others (Tice et al. 2003; Tan et al. 2004; Hsieh et al. 2005; Teh et al. 2008) can serve as a promising micro-reactor for biological and chemical assays. It employs pressure-driven flow to inject aqueous solutions into aqueous immiscible solutions and form picoliter microdroplets. The biological or chemical reagents are all encapsulated in microdroplets and each droplet is isolated by the immiscible liquid (e.g. mineral oil), thus greatly reducing sample contamination on the microchannel side walls and eliminating reagent dispersion problems. The system is useful not only for DNA sample identification, but also for quantitative analysis. The liquid-liquid reaction rate of DNA hybridization in homogeneous liquid is about 40 -fold faster than the hybridization rate in a solid-liquid interface (Gao et al. 2006). Hence, it is advantageous to improve the DNA sample/sensing probe hybridization rate using this technique rather than the conventional immobilized-probe approach. Compared to conventional microfluidics with continuous laminar flows, the microdroplet allows rapid mixing among reagents in droplet (Tice et al. 2003). The microdroplet formation system that we developed is capable of generating over five hundred monodisperse droplets per second with a size deviation of less than $2 \%$ (Tan et al. 2004). However, because of the fundamental differences between the microdroplet generation system and other conventional techniques, a liquid-liquid reaction based DNA sensing probe is needed to conduct DNA detection in microdroplets.

The distinctive biomolecular recognition and signal transduction capabilities of molecular beacons (MB) (Tyagi and Kramer 1996) have increasingly been applied to many medical diagnosis applications (Chen and Kwok 1997; Piatek et al. 1998) and biological assays, including genotyping for SNP (Kwok 2001; Syvanen 2001), multiplex genetic analysis (Marras et al. 1999; Vet et al. 1999), quantitative PCR (Vogelstein and Kinzler 1999; Chen et al. 2000), enzymatic reaction of proteins (Fang et al. 2000; Tung et al. 2000), and detection of mRNA in living cells (Sokol et al. 1998; Tsuji et al. 2000; Fang et al. 2002). MB is a class of fluorescence resonance energy transfer (FRET) molecules that are synthesized in a hairpin structure with stem and loop portions. The closed hairpin structure brings the donor fluorophor and quencher in close proximity to one another and thus quenches fluorescence. When the MB probes encounter complementary target DNA, the hairpin structure will open and the fluorescence is restored. The FRET mechanism is based on the nonradiative transfer of excitation from a donor fluorophor to an acceptor, and thus depends on the distance between the donor and acceptor, their relative orientation, and donor excited state lifetime. These outstanding properties enable FRET to be a sensitive label-free DNA detection probe in biological systems (Stryer and Haugland 1967; Dosremedios and Moens 1995; Deniz et al. 1999). The transition process between quenched and fluorescent states allows observation of bound and unbound target nucleic acids (Bonnet et al. 1999).

In order to achieve rapid DNA detection in homogeneous liquid phase with single nucleotide mismatch sensitivities, we used MB as the DNA sensing probe in the microdroplets. The target DNA and MB were all encapsulated in monodispersed picoliter droplet emulsions to reduce sample volume and to enhance the DNA detection efficiency. In this paper, we demonstrate a fast DNA sample and mutant detection in a few seconds, and evaluate dynamic MB-DNA duplex formation using label-free DNA analysis in microdroplet (LFDAM) system.

\section{Materials and results}

\subsection{Wild and mutant DNA samples}

It has been shown that females with abnormalities on BRCA1 and BRCA2 genes are prone to breast cancer and ovarian cancer (Shattuck-Eidens et al. 1995). The BRCA1 gene was chosen to demonstrate the rapid label-free DNA detection in the microdroplet platform. The target DNA, SynBRCA1, used in this research has the middle 21 nucleotides of the sequence of wild type BRCA1 gene. The sequence of SynBRCA1 is listed in Table 1. The MB-BRCA1 is the molecular beacon probe designed to detect SynBRCA1 existence in solution. The loop portion of MB-BRCA1 has a complementary sequence to SynBRCA1. MB-BRCA1 was synthesized with Cy3 labeled at its $5^{\prime}$-terminus as the fluorophor and black-hole-quencher2 
Table 1 The sequences of designed molecular beacon probes, target oligonucleotides, mutants, NS-DNA

\begin{tabular}{ll}
\hline Name & Sequence \\
\hline MB-BRCA1 & $5^{\prime}$-Cy3-CCTAGCC-CCTATGTATGC \\
& TCTTTGTTGT-GGCTAGG-BHQ2-3' \\
\cline { 2 - 2 } SynBCA1 & $5^{\prime}$-TAAC-ACAACAAAGAGCATACATAGG-GTTT-3' \\
SNP-M & $5^{\prime}$-TAAC-ACAACAAAGAaCATACATAGG-GTTT-3' \\
SNP-E & $5^{\prime}$-TAAC-ACAACAAAGAGCATACATgGG-GTTT-3' \\
2NP-M & $5^{\prime}$-TAAC-ACAACAAAGAatATACATAGG-GTTT-3' \\
2NP-ME & $5^{\prime}$-TAAC-ACAACAAAGAaCATACATgGG-GTTT-3' \\
3NP-M & $5^{\prime}$-TAAC-ACAACAAAGgatATACATAGG-GTTT-3' \\
HepCV & $5^{\prime}$-AA-GGTCGTCCTGGCAATTCCGGT-CC-3' \\
MB-HepCV & $5^{\prime}$-FAM-CCGAGC-ACCGGAATTGC \\
& CAGGACGACC-GCTCGG-BHQ1-3' \\
\hline
\end{tabular}

(BHQ2) coupled to its $3^{\prime}$ terminus as the quencher for the FRET effect as shown in Fig. 1.

In order to have sensitive and selective MB probes for hybridization analyses, the designed $\mathrm{MB}$ is required to have a high signal-to-noise ratio (SNR). SNR was calculated as

$\operatorname{SNR}=\frac{\left(I_{\mathrm{o}}-I_{\mathrm{b}}\right)}{\left(I_{\mathrm{c}}-I_{\mathrm{b}}\right)}$,

where $I_{\mathrm{b}}, I_{\mathrm{o}}$, and $I_{\mathrm{c}}$ correspond to the intrinsic fluorescence of $\mathrm{MB}$ in a buffer solution alone (background), fluorescence response of a $\mathrm{MB}$ in the presence (open-state) or in the absence (close-state) of the DNA target, respectively. BHQ2 is a novel quencher which can greatly reduce the fluorescence from $\mathrm{Cy} 3$, and thus a higher SNR value can be reached. The metal ion concentrations and the $\mathrm{pH}$ in working buffer for MB and nucleic acids hybridization will affect the optimal SNR of MB. In addition, the charge and radius of metal ions affects the stabilization of the newly formed MB-DNA duplex (Yao and Tan 2004). In order to have strong, sensitive and accurate fluorescence signals to observe the fluorescence change of MB during nucleic acid

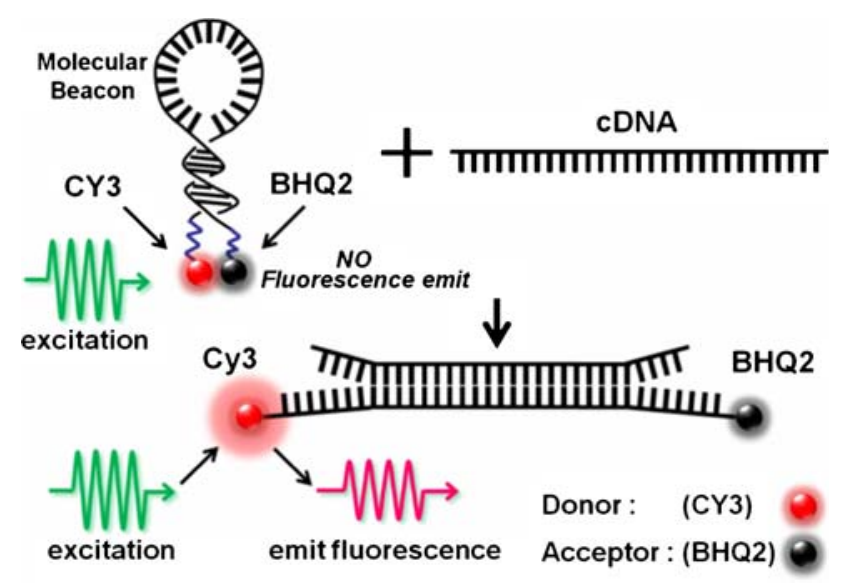

Fig. 1 Molecular beacon will emit fluorescence once it meets the complimentary DNA of the loop sequence hybridization process, the SNR of MB-BRCA1 had to be optimized to achieve maximum value. The SNR of MB-BRCA1 after hybridizing with SynBRCA1 (cDNA) was optimized by adjusting the $\mathrm{pH}$ and metal ion concentrations (Tyagi and Kramer 1996). The optimized buffer conditions for MB-BRCA1 and SynBRCA1 are $10 \mathrm{mM}$ Tris- $\mathrm{HCl}, 5 \mathrm{mM} \mathrm{MgCl}$, and $50 \mathrm{mM} \mathrm{KCl}$ (Sigma-Aldrich, $\mathrm{MO})$ in $\mathrm{pH} 8.3$ at room temperature. All working buffers used in this research were implemented in this optimized condition, if not explicitly mentioned.

As listed in Table 1, seven different sequences of oligonucleotides were synthesized as DNA samples for evaluating the selectivity and sensitivity of the designed molecular beacon probe. The SynBRCA1 is the complementary ssDNA (cDNA) for designed molecular beacon probe (MBBRCA1). Strands with SNP, double nucleotide polymorphism's (2NP), and triple nucleotide polymorphism's (3NP), have been synthesized to understand how the number of mutations affects hybridization kinetics. To further evaluate the influence of the location of mutation points on the nucleic acid, two different SNPs and 2NPs were synthesized. SNP-M and SNP-E stand for the mutation at the middle and end of SynBRCA1 sequence respectively. The sequence of 2NP-M has both mutations at the middle, and the sequence of 2NP-ME has one point mutation in the middle and the other at the end of the SynBRCA1 sequence. The 3NP has all three mutations at the middle of the SynBRCA1 sequence. Nonspecific ssDNA (NS-DNA), HepCV, is 25 nucleotides long, and has a portion of the wild type Hepatitis $\mathrm{C}$ virus gene sequence. All oligonucleotides were acquired from Integrated DNA Technologies, Inc (Coralville, IA).

\subsection{Reagents and solutions}

Mineral oil (Fisher Scientific, NH) used in this experiment contains $0.5 \%$ of Sorbitan Monooleate (Sigma-Aldrich, MO). Fluo-4 (Molecular Probes, CA) and $\mathrm{CaCl}_{2}$ (Fisher Scientific, $\mathrm{NH}$ ) were used to verify the completion of the 
reagent mixing time point in the droplets. Fluo- 4 is a $\mathrm{Ca}^{2+}$ indicator and emits fluorescence once it meets $\mathrm{Ca}^{2+}$ ions(Gee et al. 2000). Therefore, it is used to verify mixing completion inside droplets. All water used during the experiment was purified by NANOpure Water System (Thermo Fisher Scientific, MA) with a water resistance higher than $18.2 \mathrm{M} \Omega-\mathrm{cm}$ and autoclaved. All buffer components were nuclease-free, and all dilutions and centrifuge microtubes were also purified by autoclaved, nuclease-free water.

\subsection{Digital microfluidic device}

The microfluidic channel was fabricated in polydimethylsiloxane (PDMS) elastomer (Sylgard 184 from Dow Corning, Midland, MI) using the soft-photolithography technique (Duffy et al. 1998). PDMS has excellent transparent optical properties and does not auto-fluoresce as most plastics do (Duffy et al. 1998), making it an ideal material for fluorescence analysis platforms involving biochemical reactions. The PDMS microchannel was bonded to a microscope cover slide after surface treatment of oxygen plasma. The reagents and samples were driven into the microchannel via polymer tubing (Tygon Microbore, $\mathrm{OH}$ ) and nanoliter precision syringe pumps (Harvard Apparatus, MA).

The schematic layout of the microfluidic droplet system for label-free DNA analysis is shown as Fig. 2. "A" is the inlet of DNA samples, e.g. SynBRCA1, SNP-M, etc., and " $\mathrm{B}$ " is the inlet of sensing probe, e.g. MB-BRCA1. " $\mathrm{C}$ " is the inlet of aqueous immiscible continuous phase, which was mineral oil in this research. " $D$ " is the narrow multimicrochannel design to filter out PDMS debris or impurities. "E" is the break-off point, where the two merged laminar streams from A and B are broken off by shear forces from mineral oil to form microdroplets. "F" is the rapid mixing region; the $\mathrm{U}$ shape channel with sawtooth edges is designed to accelerate the mixing efficiency of reagents inside microdroplets. " $G$ " is the distance marker which is used to indicate the location of droplets in the microchannel. The width and height of the microchannel in the mixing region are 50 and $75 \mu \mathrm{m}$ respectively. The channel width was increased from 50 to $120 \mu \mathrm{m}$ after the

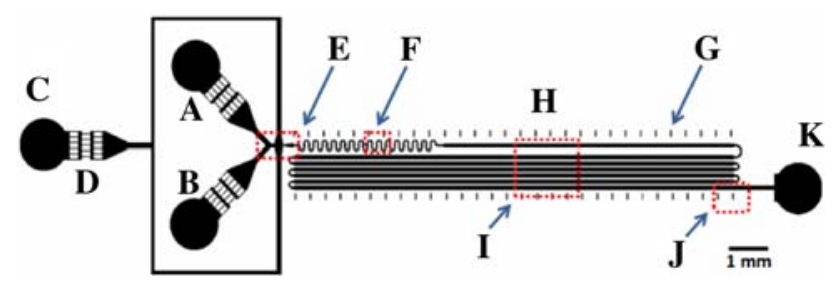

Fig. 2 Schematic of monodispersed picoliter emulsions generation device with mixing and hybridization regions mixing region to reduce the droplet's moving speed. By doing so, the fluorescence intensity during signal acquisition can increase. " $\mathrm{H}$ " is the MB and sample ssDNA hybridization region, and " $\mathrm{K}$ " is the outlet of all fluids. The total length of microchannel from mixing region to outlet is $94.5 \mathrm{~mm}$.

\subsection{Instrumentation}

We used a monochrome high-resolution deep cooled camera (Orcaerga, Hamamatsu USA, CA) as the fluorescence detector. Another monochrome high-speed camera system (Photron USA, CA), used for observing microdroplet generation and measuring droplet velocity, was also mounted to the Olympus inverted microscope system (IX51, Olympus USA, CA). The band pass filters sets, i.e. excitation filters, dichroic beam splitters, and long-pass emission filters, for both $\mathrm{Cy} 3$ and FAM fluorescence detection were all purchased from Chroma (Rockingham, VT). ImagePro Plus software (MediaCybernetics Inc., MD) was used for fluorescence data analysis.

\section{Results and discussion}

The two streams from inlets A and B merge into the main stream, which is then cut off by shear forces from the side oil streams, resulting in the formation of monodisperse picoliter droplets at point E of Fig. 2, and it is shows in Fig. 3a. The flow rates of the DNA sample, MB and mineral oil are 1.6, 1.6 and $1.5 \mu \mathrm{l} / \mathrm{min}$ respectively. The DNA samples and MB are encapsulated inside the microdroplet, and while the microdroplet is traveling in the sawtooth edged serpentine channel configuration (Fig. 3b), chaotic advection flow occurs within the droplet (Song and Ismagilov 2003; Liau et al. 2005; Muradoglu and Stone 2005) and allows the DNA sample and MB-BRCA1 to mix rapidly. In Fig. 3c, we can see the droplets traveling in the "hybridization" region (I region in Fig. 2) where MB and DNA samples interact and bind to each other. The microdroplet generation rate was measured by the high-speed camera to be 377 droplets/s. The droplets are monodispersed, and the size deviation is less than 2\%, as reported (Tan et al. 2006). The droplets are nominally $64 \mu \mathrm{m}$ in diameter, or 137 picoliters in volume. In Fig. 3d, it illustrates the concepts of LFDAM system. The the DNA and MB were first encapsulated inside droplets then allowed to rapidly mix together. MB restores fluorescence after hybridizing with cDNA, and the fluorescence emits from MB was gradually increasing from the upstream to the downstream.

In order to verify completion of reagent mixing in droplets, the rapid fluorescent reaction of Fluo-4 $(50 \mu \mathrm{M})$ and $\mathrm{Ca}^{2+}(150 \mu \mathrm{M})$ was used as an indicator under the 
Fig. 3 The merged A and B streams are broken off by side streams and form microdroplets. a Consecutive micrographs of droplet generation. The generation rate is $2.66 \mathrm{~ms} /$ droplet. b DNA and $\mathrm{MB}$ are rapidly mixed in sawtooth edged U-shaped channel. c Picoliter droplets moving in hybridization region. The area of image is indicated as $I$ in Fig. 2. d The DNA and MB were first encapsulated inside droplets then allowed to rapidly mix together. MB restores fluorescence after hybridizing with cDNA
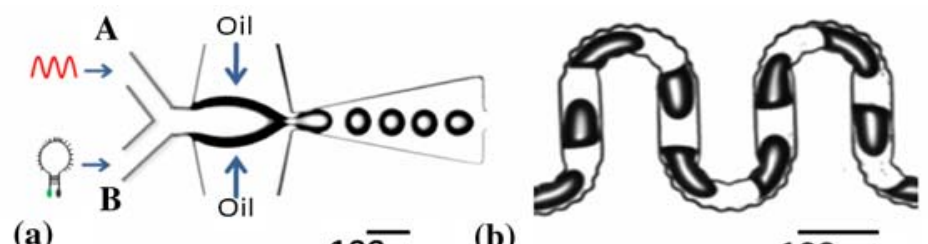

$\overline{100 \mu \mathrm{m}}$

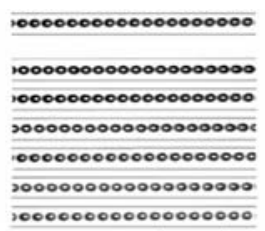

(c)

$3 \overline{300 \mu \mathrm{m}}$

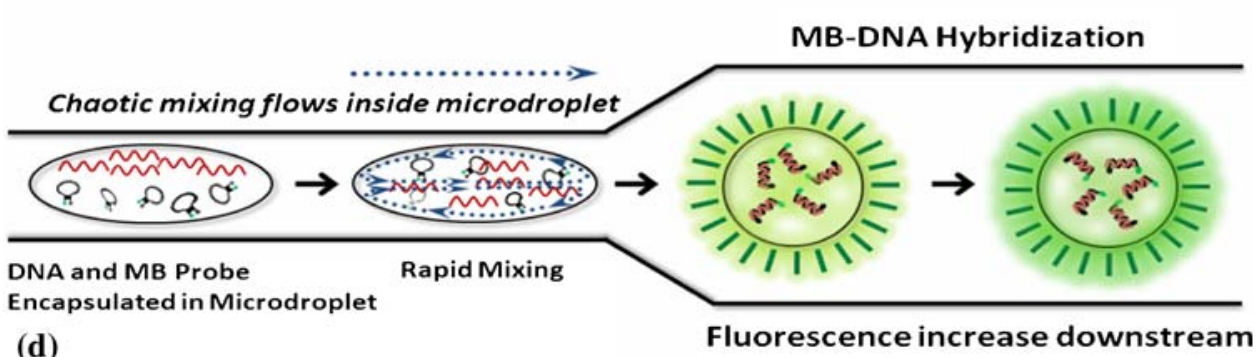

(d)

same flow rate conditions. Once Fluo- 4 meets $\mathrm{Ca}^{2+}$, it will emit strong fluorescence. This reaction time is less than a few microseconds (Gee et al. 2000) and thus negligible. The fluorescence intensity achieves a maximum and remains constant once reagents are thoroughly mixed. The mixing completion time in the droplet is $T_{\mathrm{m}}=L_{\mathrm{m}} / V_{\mathrm{m}}$, where $L_{\mathrm{m}}$ is the traveled distance of the droplet from the break-off point and $V_{\mathrm{m}}$ is droplet velocity measured in the mixing region. As show in Fig. $4 \mathrm{a}-\mathrm{c}$, after the tenth turn of the sawtooth edged microchannel, the Fluo-4 fluorescence saturated and remained constant thereafter. Since $L_{\mathrm{m}}$ is $1,835 \mu \mathrm{m}$, and $V_{\mathrm{m}}$ is $21,094 \mu \mathrm{m} \mathrm{s}^{-1}$, the mixing completion time $T_{\mathrm{m}}$ was calculated to be $87 \mathrm{~ms}$. Thus, we designate this location as the starting point of DNA sample-MB duplex formation or DNA sample-MB hybridization.

\subsection{MB-DNA duplex formation evaluation}

The DNA sample and MB-BRCA1 were injected into the microchannel, with concentrations of 2.5 and $1 \mu \mathrm{M}$ respectively. Due to the monodispersity of microfluidic droplet generation, each droplet encapsulated the same number of MB and DNA. By accumulating fluorescence at one location, the fluorescence signal emitted from MB can be amplified using a longer exposure time without worrying about the change of different reaction stages. By recording fluorescence signal downstream of microchannel, MB-DNA duplex formation reaction can be observed at different stages. Droplets carrying MB-DNA duplex formation reaction stages are "captured by repeated images" and "frozen" along the microchannel.

The MB-DNA duplex formation time was converted to travelled distance. Therefore, the acquisition of reaction signals is no longer time-dependent at one location. In other words, it is time-resolved. As illustrated in Fig. 5a, the MB-DNA duplex formation time, $T_{\mathrm{h}}$ is acquired from $T_{\mathrm{h}}=L_{\mathrm{h}} / V_{\mathrm{h}}$, where $L_{\mathrm{h}}$ is the travel distance of the droplet from the mixing completion point and $V_{\mathrm{h}}$ is the droplet velocity in hybridization region. The velocity $V_{\mathrm{h}}$ is measured to be $8,723 \mu \mathrm{m} \mathrm{s}^{-1}$. It is important to note that because this system does not have to record the fluorescence change in time, it is not limited by the sensitivity and shutter speed of the sensor. The width of the observing window is $2,175 \mu \mathrm{m}$, and the droplet velocity is $8,723 \mu \mathrm{m} \mathrm{s}^{-1}$. Therefore, the droplet is only exposed to the excitation for $249 \mathrm{~ms}$ under a $4 \times$ magnification. The fluorescence image taken by the CCD sensor has $3.24 \mu \mathrm{m} /$ pixel resolution for $4 \times$ magnification. The fluorescence image was later analyzed by ImagePro to measure
Fig. 4 Fluo-4 was used to verify the mixing completion inside droplets. a Fluorescent image of the emulsification region. b Fluorescence of Fluo4 was not yet saturated at 4 th mixing turn. $\mathbf{c}$ The fluorescence of Fluo-4 fills the whole droplet at the tenth turn, indicating completion of mixing
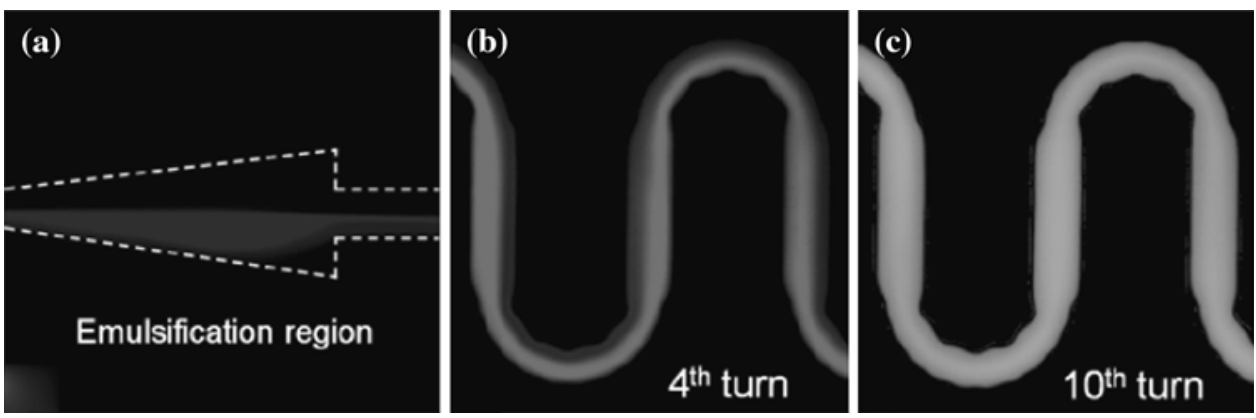
(a)

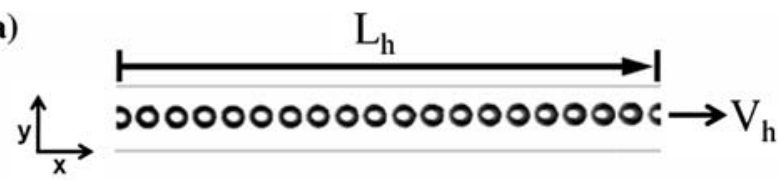

(b)

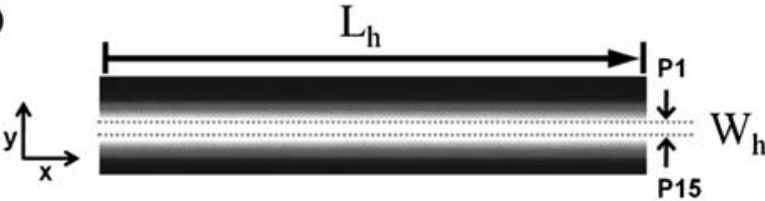

Fig. 5 MB hybridizes with targets and emits fluorescence, which was analyzed in LFDAM. a Time-resolved picoliter droplets moving in microchannel. The coordinate reaction time can be acquired from the droplet location in the microchannel. b MB fluorescence signal in microdroplet was analyzed using vertical average-multiple-line-scan method

fluorescence intensity. One data point equals $0.37 \mathrm{~ms}$ of the signal in this measurement.

From the high-speed camera measurement, we found there were 452 droplets repeated at the observation point in $1.2 \mathrm{~s}$. Therefore, the fluorescence signal of $\mathrm{MB}$ in the droplets was amplified 452 folds in $1.2 \mathrm{~s}$, as compared to only one passing fluorescent droplet. This approach can increase statistical significance; one datum represents hundreds of micro assays, and reduces assay-to-assay deviation. The fluorescence in Fig. 6a was analyzed using the vertical average-multiple-line-scan method. Each data point shown in Fig. 5b was taken as an average of 15 horizontal ( $x$-axis) data pixels, $W_{\mathrm{v}}=48 \mu \mathrm{m}$, those at the same $y$-axis location. It shows fluorescence in the channel gradually increasing from the upstream to the downstream; MB gradually restores fluorescence during the

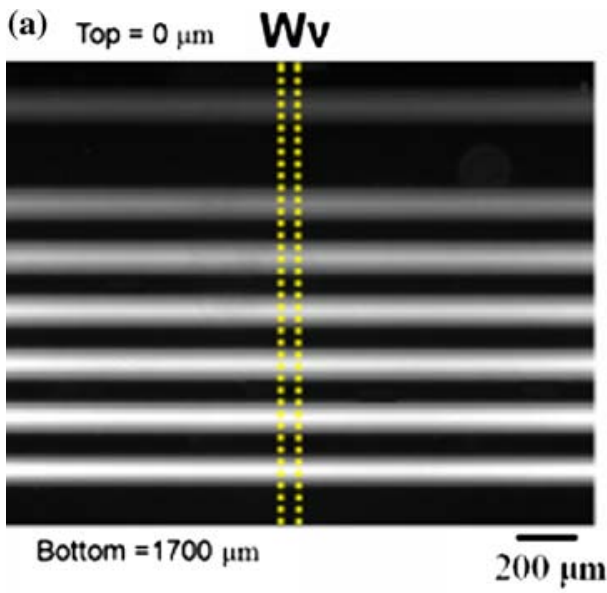

Fig. 6 The dynamic binding reaction of target DNA with MB can be observed from the fluorescence change of MB inside droplet. a The horizontal average-multiple-line-scan method is used to measure the fluorescence intensity. The yellow dash line represents the hybridization process. The " $g$ " shown in the Fig. $6 \mathrm{~b}$ is the gap between microchannels and it is equal to $70 \mu \mathrm{m}$. The " $d$ " is the diameter of microdroplet, and it is equal to $64 \mu \mathrm{m}$. The " $w$ " is the width of microchannel in hybridization region, and it is equal to $120 \mu \mathrm{m}$. We conduct the time-resolved fluorescence measurement with horizontal average-multiple-line-scan method. Each data point shown in Fig. 7 was taken as an average of 15 vertical $\left(y\right.$-axis) data pixels, $W_{\mathrm{h}}=48 \mu \mathrm{m}$, those at the same $x$-axis location, from P1 (top pixel) to P15 (bottom pixel), as shown on Fig. $5 b$. The total width of 15 pixels under $4 \times$ magnifications is equal to $48 \mu \mathrm{m}$; it covers about $75 \%$ of the droplet width which is $W_{\mathrm{h}}$ as shown in Fig. 6b. We took a total of seven fluorescent area images to cover the whole mixing and hybridization regions of the device. Each area image was taken three times $(n=3)$, and all fluorescence images were normalized by fluorescence analysis tools (ImagePro) before signals were analyzed.

The fluorescence restoration process of $\mathrm{MB}$ during the duplex formation with different cDNA or mutants in microdroplet is shown in Fig. 7. The hybridization signals at the channel turns were not collected. However, the $23 \mathrm{~ms}$ of time lapse in each turn was counted. The time axis covers from the mixing completion point to the end of hybridization region just before the outlet reservoir. The fluorescence reaches a plateau in Region 3. The fluorescence intensity from $\mathrm{MB}$ remains constant at this plateau region, representing the completion of MB-DNA duplex formation. Therefore, the LFDAM device used in this research can evaluate the dynamic hybridization kinetics in less than $10 \mathrm{~s}$. Faster detection rates can be reached with higher DNA concentrations.

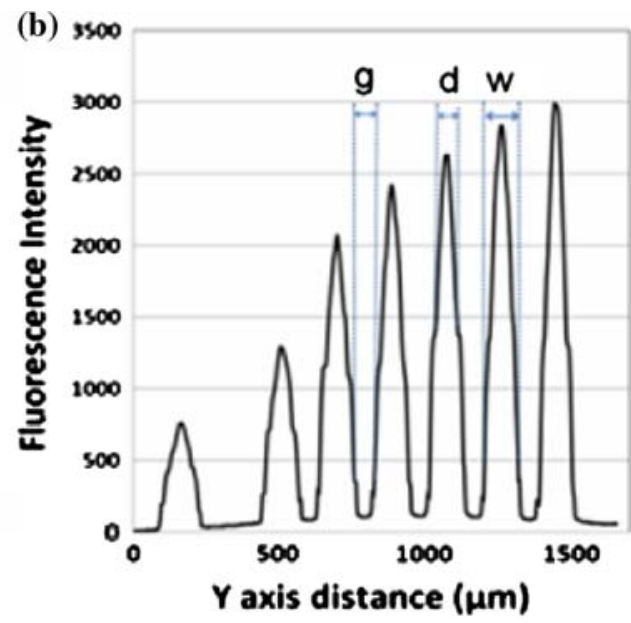

fluorescence analysis from the top to the bottom of an image. The width of line scan is $\mathrm{Wv}=48 \mu \mathrm{m}$. The image is from region I in Fig. 2. b The measured result shows that the MB fluorescence gradually increases downstream in the LFDAM 


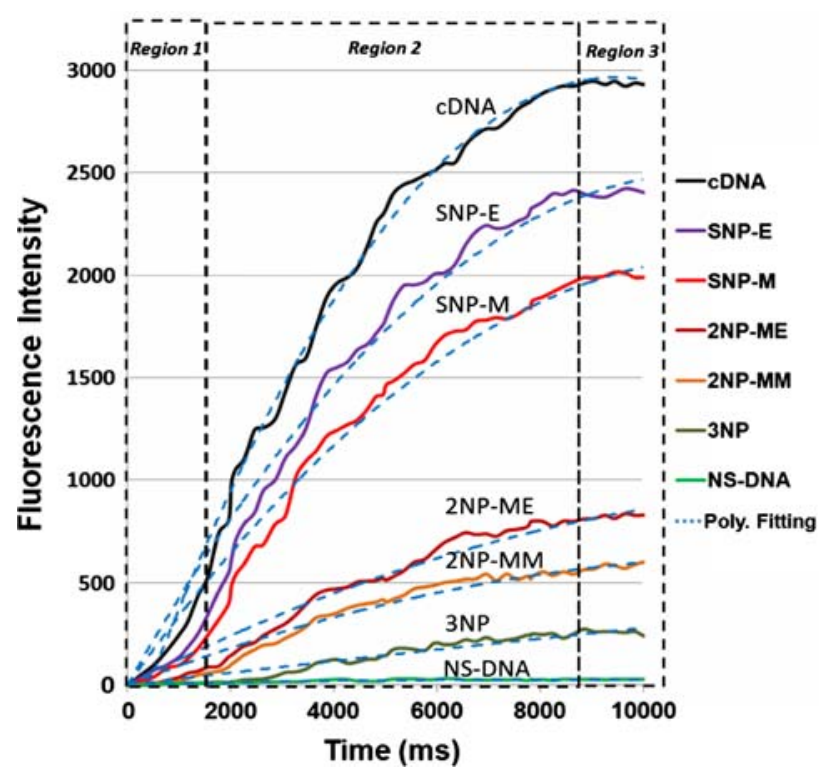

Fig. 7 Comparison of fluorescence intensity change of MB with different ssDNA sequences during hybridization in the microdroplets. The dashed-lines are second-order best-fitting curves of the DNA samples

Photobleaching of fluorophors that are labeled on DNA has been an issue in many fluorescence analyses; it typically occurs in a matter of only few microseconds to seconds (Osborne et al. 2001; Yao et al. 2003). Specifically for $\mathrm{Cy} 3$, photobleaching manifests in a few seconds (Eggeling et al. 1998). In this experiment, the MB passes the $4 \times$ magnification observation window as quickly as $249 \mathrm{~ms}$. The time-resolve fluorescence analysis method not only reduces the photobleaching problem while monitoring the real-time reaction, but it can also compensate for the short exposure of each droplet by accumulating repeating fluorescent microdroplets at the same location. In this timeresolved approach, the fidelity of fluorescence signals can be retained.

We have also compared the reaction of MB with different mutants of SynBRCA1 samples as well as nonspecific DNA. The sequences of all synthetic ssDNA samples are listed in Table 1. In Fig. 7, the measured results of different mutants are compared and it is found that the designed MB-BRCA1 is very selective and capable of distinguishing all six different mutant ssDNAs, as well as the SNP samples. The SNR values shown in Fig. 8 were acquired from the average fluorescence intensities of each ssDNA in region 3 of Fig. 7, and calculated by Eq. 1. MB with cDNA has a SNR as high as 128. The designed MBBRCA1 is very selective in discriminating among the SNP. Moreover, it is able to discriminate the point mutation in the middle (SNP-M) or at the end (SNP-E) of the sequence. The SNR of SNP-M is $17 \%$ smaller than SNP-E. Therefore, a point mutation on the middle of SNP hinders the

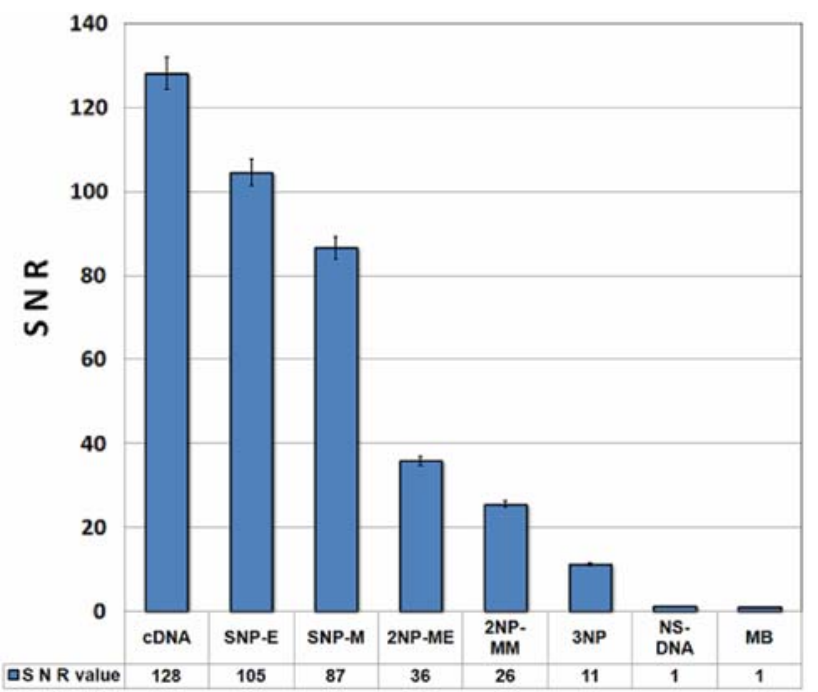

Fig. 8 The SNR values of MB with different samples, i.e. cDNA, mutated cDNAs, and NS-DNA

MB-DNA hybridization more than one at the end of sequence. Also, the SNR of 2NP-MM is $28 \%$ smaller than that of 2NP-ME. The difference between SNP-M and SNP-E is smaller than the difference between 2NP-MM and 2NP-ME. On the other hand, the SNR of 2NP-MM is $70 \%$ smaller than SNP-M. The SNR of 3NP is $58 \%$ smaller than 2NP-MM. Thus, increasing the point mutation number will reduce the SNR and adversely influence the stability and formation of MB-DNA duplex. We can therefore assume that the mutation at the middle portion has a larger affect on reducing the ability for MB to open its hairpin structure. In Fig. 7, it was also observed that the dynamic hybridization kinetics of MB-BRCA1 with SNP-M and SNP-E are closer to cDNA. The kinetics for 2NP-MM and 2NP-ME are more similar to $3 \mathrm{NP}$ than SNP. Since MB does not hybridize with NS-DNA, the MB doesn't restore fluorescence and thus the SNR of non-specific DNA is close to one. The SNR of 3NP is 11 times higher than that of NS-DNA. These results show that using the microfluidic device with designed MB-BRCA1 allows for rapid and easy discrimination of DNA with different mutation points in addition to dynamic hybridization within a few seconds. It is a promising tool to further develop for genotyping assays (Dittrich and Manz 2006).

\subsection{Dual DNAs detection in droplet}

Dual DNAs detection in the microfluidic device was further investigated. $2.5 \mu \mathrm{M}$ of SynBRCA1 and HepCV were mixed and injected into microchannel via inlet $A$, and $1 \mu \mathrm{m}$ of MB-BRCA1 and MB-HepCV were mixed and injected into the microchannel via inlet $\mathrm{B}$. The sequence of MB-HepCV is listed in Table 1. SynBRCA1, HepCV, 


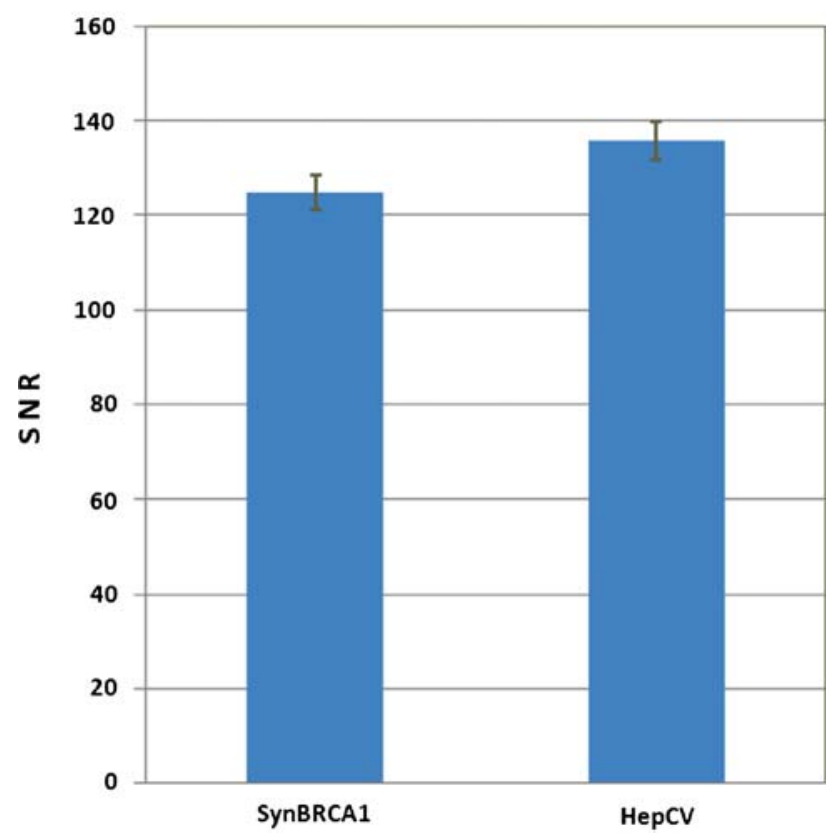

Fig. 9 The measured SNRs of two different MBs (MB-BRCA1 and MB-HepCV) with their cDNAs (SynBRCA1 and HepCV) in microdroplets using LFDAM system

MB-BRCA1, and MB-HepCV were all encapsulated in microdroplet and rapidly mixed. The emitting fluorescence from microdroplet includes $\mathrm{Cy} 3$ from MB-BRCA1 and FAM from MB-HepCV. The fluorescence was measured by specific filter sets with different band pass for $\mathrm{Cy} 3$ or FAM. As shown in Fig. 8, the HepCV (NS-DNA) did not interact with MB-BRCA1 with SNR equal to background of MB-BRCA1. Figure 9 shows that the measured $\mathrm{Cy} 3$ and FAM fluorescence from MB-BRCA1 and MB-HepCV in microdroplet has SNR 125, and 136, respectively.

\subsection{Dynamic range of linearity}

The sensitivity of this label-free DNA detection in picoliter droplet system was characterized by analyzing different concentrations of SynBRCA1. Different concentrations $(125,250$ and $500 \mathrm{nM}, 1,1.5$ and $2 \mu \mathrm{M})$ of SynBRCA1 and $2 \mu \mathrm{M}$ of $\mathrm{MB}$ were encapsulated in each microdroplet. The MB-BRCA1 restored fluorescence after it fully hybridized with SynBRCA1. The SynBRCA1 detection by MB-BRCA1 was conducted by taking fluorescence signal at the end of hybridization region ("J" in Fig. 2) with $1.2 \mathrm{~s}$ of fluorescence acquisition. As shown in Fig. 10, $2 \mu \mathrm{m}$ SynBRCA1 has SNR as high as 125 . The best-fitting curve (dashed-line) shows a strongly linear relation between SNR and DNA concentration from $125 \mathrm{nM}$ to $2 \mu \mathrm{M}$. Detection of DNA concentrations above MB concentration $(2 \mu \mathrm{M}$ in this study) is possible but highly non-linear. On the other hand, a signal of DNA concentration below $125 \mathrm{nM}$ was

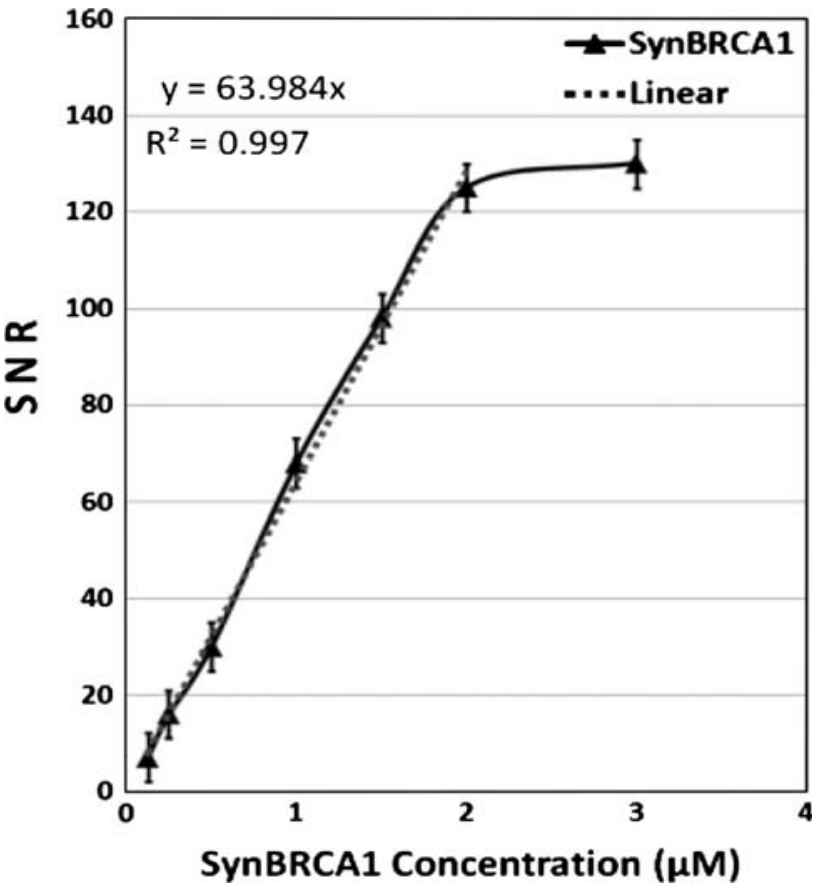

Fig. 10 Quantitative analysis of the cDNA-SynBRCA1 in LFDAM has a linear dynamic range from $125 \mathrm{nM}$ to $2 \mu \mathrm{M}$ when $\mathrm{MB}$ is kept at $2 \mu \mathrm{M}$. The solid-line is the SNR value of different concentrations of SynBRCA1. The dashed-line is the linear fitting curve for the SNR of different SynBRCA1 concentrations. This linear range is highly dependent on device sensitivity and MB concentration

insignificant when compared to the noise of MB $(2 \mu \mathrm{M})$. Therefore, detection below $125 \mathrm{nM}$ could only be accomplished if a lower concentration of MB was used. $500 \mathrm{fM}$ of DNA was detected when $2.5 \mathrm{nM} \mathrm{MB}$ was used. These results suggest that the use of $\mathrm{MB}$ in the microfluidic device can rapidly (within $10 \mathrm{~s}$ ) detect not only presence, but also concentration of target DNAs. An even faster detection may be achieved since hybridization in region 1 of Fig. 7 is already distinguishable among different DNA samples.

The folding-unfolding transitions of the hairpin-structured molecular beacon (MB) are dependent on the following parameters: the length of the loop portion, the loop sequence, and the activation energies of the openclose transitions. The conformational fluctuation kinetics of the DNA hairpin-loop are very fast, and the opening and closing transition in the MB has been reported in the microseconds range (Bonnet et al. 1998). In the beginning of region $1, \mathrm{MB}$ is in the closed state, and $\mathrm{MB}$ and target DNA are in close proximity to each other. As the basepairing increases in number, the intermolecular forces increase as well. As soon as the intermolecular forces become larger than the intramolecular forces at the stem, the hairpin structure starts to open and frees the fluorescence from the fluorophor. As the base-pairing process 
continues, the distance of donor fluorophor and acceptor fluorophor (quencher) gradually increases, and the fluorescence is restored while the FRET phenomenon ceases (Stryer 1978; Jares-Erijman and Jovin 2003). Instability of the hairpin structure of the MB-DNA duplex induces the association or dissociation transmission during this stage of the hybridization process. The MB-DNA duplex formation can be considered to have the open/close conformational fluctuation of a typical of hairpin MB fluorescence restoring (Bonnet et al. 1998; Yao et al. 2003). The open/close fluctuation rate of MB-DNA varied with different sequences of DNA. The MB-DNA duplex has several cycles of conformational changes in open and close states. The more stable the MB-DNA duplex, the stronger the MB fluorescence restored. The measured fluorescence in droplet can be expressed as Eq. 2.

$F=k(C \times V \times f \times T) \frac{\left[\left(N \times I_{\mathrm{o}}\right)+[(t-N)] \times I_{\mathrm{c}}\right]}{t}$

$F$ is the total fluorescence intensity measured, $I_{\mathrm{c}}$ is the background $\mathrm{MB}$ fluorescence in its closed state, $I_{\mathrm{o}}$ is the restored fluorescence in its open state (von Ahsen et al. 1999), $N$ is the time when MB is in open state, and $(t-N)$ is the time MB is in closed state. $C$ is the concentration of complementary DNA under the assumption that the concentration of MB is higher than the sample. $V$ is the volume of droplet and $f$ is the number of droplets passing through the observation window in 1-s. $T$ is the total CCD sensor data collection time and $t$ is the time for a droplet to pass one sensor pixel of the CCD camera; $t=d / s$ where $d$ is the distance of one pixel and $s$ is the droplet traveling velocity in the hybridization region. Also, $k$ is the correction constant which is related to light intensity. Using $4 \times$ magnification and fluorescence acquisition for $1.2 \mathrm{~s}$, one droplet will take $0.37 \mathrm{~ms}$ to pass through one pixel on the CCD sensor. Equation 2 can be rewritten as Eq. 3

$F=k\left(3.1 \times 10^{-10}\right) \frac{\left[\left(N \times I_{\mathrm{o}}\right)+[(0.37-N)] \times I_{\mathrm{c}}\right]}{0.37}$

The measured fluorescence intensity is directly proportional to sample concentration, droplet volume, droplet generation rate, and fluorescence acquisition time. The selection of donor fluorophor will affect the value of $I_{\mathrm{o}}$, and the selection of quencher will affect the value of $I_{\mathrm{c}}$. The value of $N$ is inversely proportional to the number of mutation points. In other words, these results disclose the characteristic probability of MB-BRCA1 closing and opening for different mutations of SynBRCA1.

The LFDAM microfluidic device demonstrates the ability to localize MB with DNA samples in picoliter droplets and not only detect mutants in a few seconds, but also evaluate the different hybridization kinetics between mutants. It was also observed that higher concentrations of target DNA and MB probe in microdroplets cause higher rates of fluorescence increase; or, larger $\Delta f / \Delta t$ in Fig. 7, where $\Delta f$ is the fluorescence change in period of time $\Delta t$. Equation 4 describes the time required for $\mathrm{MB}$ to meet DNA in microdroplet after mixing. It can be applied by diffusion based DNA transport in solution.

$\tau=\frac{\chi^{2}}{2 D}$

$\tau$ is the time required for a molecule to diffuse over distance $\chi$, and $D$ is the diffusion coefficient of DNA. The typical value of $D$ is of $9.948 \times 10 \mathrm{E}-7 \mathrm{~cm}^{2} \mathrm{~s}^{-1}$ (for 18base ssDNA)(Nkodo et al. 2001). The diameter of the microdroplet is $64 \mu \mathrm{m}$ and volume is 137 picoliter. Since the concentration of DNA $(1.25 \mu \mathrm{M})$ is higher than MB $(0.5 \mu \mathrm{M})$, the average distance of MB and DNA is determined by DNA. The average DNA number in one droplet is $1031.3 \times 10^{5}$. If we do not consider the size of molecules, the average distance of MB and DNA is $11.9 \mathrm{~nm}$. Therefore, the time required for MB to meet DNA is approximately $0.7 \mathrm{~ms}$; thus, the diffusion time is so fast after mixing, it can be ignored.

\section{Conclusion}

We have developed a LFDAM system for using FRET molecules to rapidly detect DNA in Microdroplets and also capable evaluate the dynamic hybridization process through a time-resolved method. Because convection-driven mixing occurs in picoliter microdroplets, the often lengthy diffusion time in bulk solution or non-droplet microfluidic channels is greatly reduced. Consequently, the reoccurring identical droplets represent the hybridization stage via fluorescent signals, and the different reaction stages in droplets were shown at different traveling distances. The reaction time correlates to the distance traveled by the droplets in the microchannel. Since each droplet is exposed to excitation source for only $249 \mathrm{~ms}$, the photobleaching problem was reduced to a minimum. The designed MB-BRCA1 for SynBRCA1 detection can reach a SNR as high as 128 demonstrating LFDAM as a sensitive platform to distinguish SNP point mutation at various sites and elicit the difference of their hybridization kinetics. Duplex target detection in one microdroplet was demonstrated by analyzing SynBRCA1 and HepCV in LFDAM. The limitation of detection in LFDAM with MB can reach as low as $500 \mathrm{fM}$. This microdroplet array and molecular beacon based system, LFDAM, is promising for highthroughput genotyping of SNPs in pharmacogenomics research, where the identification of variations in specific genes is necessary to evaluate drug efficacy, toxicity and 
metabolism. It will be useful for establishing optimal therapeutic strategies for individual patients. LFDAM may also be used to analyze other rapid biomolecular reactions, such as enzymatic activity, multiplex nucleic acid detection, cells, RNA, and proteins folding whose analyses require small volume, rapid mixing, and minimum effect of photobleaching.

Acknowledgments The authors would like to gratefully acknowledge Yuh Adam Lin, Professor James Brody, Joseph Harris, Alan Lee, and Wajeeh Saadi of the Biomedical Engineering Department, University of California, Irvine for their precious suggestions and generosity in letting us use their lab equipments. This research was funded by Defense Advanced Research Projects Agency (DARPA) under the MF3 N/MEMS S\&T Centers (HR001106-1-0050), and NASA through the University Affiliated Research Center (NAS203144).

Open Access This article is distributed under the terms of the Creative Commons Attribution Noncommercial License which permits any noncommercial use, distribution, and reproduction in any medium, provided the original author(s) and source are credited.

\section{References}

Bonnet G, Krichevsky O et al (1998) Kinetics of conformational fluctuations in DNA hairpin-loops. Proc Natl Acad Sci USA 95(15):8602-8606

Bonnet G, Tyagi S et al (1999) Thermodynamic basis of the enhanced specificity of structured DNA probes. Proc Natl Acad Sci USA 96(11):6171-6176

Caruso F, Rodda E et al (1997) Quartz crystal microbalance study of DNA immobilization and hybridization for nucleic acid sensor development. Anal Chem 69(11):2043-2049

Chen XN, Kwok PY (1997) Template-directed dye-terminator incorporation (TDI) assay: a homogeneous DNA diagnostic method based on fluorescence resonance energy transfer. Nucleic Acids Res 25(2):347-353

Chen W, Martinez G et al (2000) Molecular beacons: a real-time polymerase chain reaction assay for detecting Salmonella. Anal Biochem 280(1):166-172

Deniz AA, Dahan M et al (1999) Single-pair fluorescence resonance energy transfer on freely diffusing molecules: observation of Forster distance dependence and subpopulations. Proc Natl Acad Sci USA 96(7):3670-3675

Dittrich PS, Manz A (2006) Lab-on-a-chip: microfluidics in drug discovery. Nat Rev Drug Discov 5(3):210-218

Dosremedios CG, Moens PDJ (1995) Fluorescence resonance energytransfer spectroscopy is a reliable ruler for measuring structuralchanges in proteins-dispelling the problem of the unknown orientation factor. J Struct Biol 115(2):175-185

Duffy DC, McDonald JC et al (1998) Rapid prototyping of microfluidic systems in poly(dimethylsiloxane). Anal Chem 70(23):4974-4984

Eggeling C, Widengren J et al (1998) Photobleaching of fluorescent dyes under conditions used for single-molecule detection: evidence of two-step photolysis. Anal Chem 70(13):2651-2659

Fang XH, Li JJ et al (2000) Using molecular beacons to probe molecular interactions between lactate dehydrogenase and single-stranded DNA. Anal Chem 72(14):3280-3285
Fang XH, Mi YM et al (2002) Molecular beacons-fluorogenic probes for living cell study. Cell Biochem Biophys 37(2):71-81

Gao Y, Wolf LK et al (2006) Secondary structure effects on DNA hybridization kinetics: a solution versus surface comparison. Nucleic Acids Res 34(11):3370-3377

Gee KR, Brown KA et al (2000) Chemical and physiological characterization of fluo- $4 \mathrm{Ca}^{2+}$-indicator dyes. Cell Calcium 27(2):97-106

He L, Musick MD et al (2000) Colloidal Au-enhanced surface plasmon resonance for ultrasensitive detection of DNA hybridization. J Am Chem Soc 122(38):9071-9077

Hsieh AT, Hung LH, Lee AP (2005) Rapid breast cancer gene detection in picoliter droplet. The 9th international conference on miniaturized systems for chemistry and life sciences, Boston

Jares-Erijman EA, Jovin TM (2003) FRET imaging. Nat Biotechnol 21(11):1387-1395

Jobs M, Fredriksson S et al (2002) Effect of oligonucleotide truncation on sing le-nucleotide distinction by solid-phase hybridization. Anal Chem 74(1):199-202

Kamholz AE, Weigl BH et al (1999) Quantitative analysis of molecular interaction in a microfluidic channel: the T-sensor. Anal Chem 71(23):5340-5347

Kwok PY (2001) Methods for genotyping single nucleotide polymorphisms. Annu Rev Genomics Hum Genet 2:235-258

Lagally ET, Medintz I et al (2001) Single-molecule DNA amplification and analysis in an integrated microfluidic device. Anal Chem 73(3):565-570

Liau A, Karnik R et al (2005) Mixing crowded biological solutions in milliseconds. Anal Chem 77(23):7618-7625

Lipshutz RJ, Fodor SPA et al (1999) High density synthetic oligonucleotide arrays. Nat Genet 21:20-24

Liu RH, Stremler MA et al (2000) Passive mixing in a threedimensional serpentine microchannel. J Microelectromech S 9(2):190-197

Livak KJ (1999) Allelic discrimination using fluorogenic probes and the 5'nuclease assay. Genet Anal-Biomol E 14(5/6):143-149

Marras SAE, Kramer FR et al (1999) Multiplex detection of singlenucleotide variations using molecular beacons. Genet Anal Biomol E 14(5/6):151-156

Muradoglu M, Stone HA (2005) Mixing in a drop moving through a serpentine channel: a computational study. Phys Fluids 17:073305

Nkodo AE, Garnier JM et al (2001) Diffusion coefficient of DNA molecules during free solution electrophoresis. Electrophoresis 22(12):2424-2432

Okahata Y, Kawase M et al (1998) Kinetic measurements of DNA hybridisation on an oligonucleotide-immobilized 27-MHz quartz crystal microbalance. Anal Chem 70(7):1288-1296

Osborne MA, Barnes CL et al (2001) Probing DNA surface attachment and local environment using single molecule spectroscopy. J Phys Chem B 105(15):3120-3126

Peterson AW, Heaton RJ et al (2000) Kinetic control of hybridization in surface immobilized DNA monolayer films. J Am Chem Soc 122(32):7837-7838

Peterson AW, Heaton RJ et al (2001) The effect of surface probe density on DNA hybridization. Nucleic Acids Res 29(24):51635168

Piatek AS, Tyagi S et al (1998) Molecular beacon sequence analysis for detecting drug resistance in Mycobacterium tuberculosis. Nat Biotechnol 16(4):359-363

Sanders GHW, Manz A (2000) Chip-based microsystems for genomic and proteomic analysis. Trac Trends Anal Chem 19(6):364-378

Shattuck-Eidens D, Mcclure M et al (1995) A collaborative survey of 80 mutations in the BRCA1 breast and ovarian cancer susceptibility gene: implications for presymptomatic testing and screening. J Am Med Assoc 273(7):535-541 
Sia SK, Whitesides GM (2003) Microfluidic devices fabricated in poly(dimethylsiloxane) for biological studies. Electrophoresis 24(21):3563-3576

Sokol DL, Zhang XL et al (1998) Real time detection of DNA RNA hybridization in living cells. Proc Natl Acad Sci USA 95(20):11538-11543

Song H, Ismagilov RF (2003) Millisecond kinetics on a microfluidic chip using nanoliters of reagents. J Am Chem Soc 125(47):14613-14619

Steel AB, Levicky RL et al (2000) Immobilization of nucleic acids at solid surfaces: effect of oligonucleotide length on layer assembly. Biophys J 79(2):975-981

Stryer L (1978) Fluorescence energy-transfer as a spectroscopic ruler. Annu Rev Biochem 47:819-846

Stryer L, Haugland RP (1967) Energy transfer-a spectroscopic ruler. Proc Natl Acad Sci USA 58(2):719-726

Syvanen AC (2001) Accessing genetic variation: genotyping single nucleotide polymorphisms. Nat Rev Genet 2(12):930-942

Tan YC, Fisher JS et al (2004) Design of microfluidic channel geometries for the control of droplet volume, chemical concentration, and sorting. Lab Chip 4(4):292-298

Tan YC, Cristini V et al (2006) Monodispersed microfluidic droplet generation by shear focusing microfluidic device. Sens Actuators B 114(1):350-356

Teh SY, Lin R et al (2008) Droplet microfluidics. Lab Chip 8(2):198220

Thiel AJ, Frutos AG et al (1997) In situ surface plasmon resonance imaging detection of DNA hybridization to oligonucleotide arrays on gold surfaces. Anal Chem 69(24):4948-4956

Tice JD, Song $\mathrm{H}$ et al (2003) Formation of droplets and mixing in multiphase microfluidics at low values of the Reynolds and the capillary numbers. Langmuir 19(22):9127-9133
Tsuji A, Koshimoto H et al (2000) Direct observation of specific messenger RNA in a single living cell under a fluorescence microscope. Biophys J 78(6):3260-3274

Tung CH, Mahmood U et al (2000) In vivo imaging of proteolytic enzyme activity using a novel molecular reporter. Cancer Res 60(17):4953-4958

Tyagi S, Kramer FR (1996) Molecular beacons: probes that fluoresce upon hybridization. Nat Biotechnol 14(3):303-308

van't Veer LJ, Dai HY, et al (2002) Gene expression profiling predicts clinical outcome of breast cancer. Nature 415(6871):530-536

Vet JAM, Majithia AR et al (1999) Multiplex detection of four pathogenic retroviruses using molecular beacons. Proc Natl Acad Sci USA 96(11):6394-6399

Vogelstein B, Kinzler KW (1999) Digital PCR. Proc Natl Acad Sci USA 96(16):9236-9241

von Ahsen N, Oellerich M et al (1999) Application of a thermodynamic nearest-neighbor model to estimate nucleic acid stability and optimize probe design: Prediction of melting points of multiple mutations of apolipoprotein B-3500 and factor $\mathrm{V}$ with a hybridization probe genotyping assay on the LightCycler. Clin Chem 45(12):2094-2101

Wong ELS, Chow E et al (2005) DNA recognition interfaces: the influence of interfacial design on the efficiency and kinetics of hybridization. Langmuir 21(15):6957-6965

Yao G, Tan WH (2004) Molecular-beacon-based array for sensitive DNA analysis. Anal Biochem 331(2):216-223

Yao G, Fang XH et al (2003) Monitoring molecular beacon DNA probe hybridization at the single-molecule level. Chem Eur J 9(22):5686-5692 